\title{
EUROfusion
}

\section{Pattern recognition in nuclear fusion data by means of geometric methods in probabilistic spaces}

\section{Geert Verdoolaege}

Department of Applied Physics, Ghent University, Ghent, Belgium

Laboratory for Plasma Physics, Royal Military Academy (LPP-ERM/KMS), Brussels, Belgium

ECEA 2017, November 21 - December 1, 2017

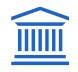

GHENT UNIVERSITY

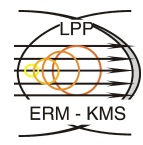

This work has been carried out within the framework of the EUROfusion Consortium and has received funding from the

Euratom research and training programme 2014-2018 under grant agreement No 633053. The views and opinions expressed herein do not necessarily reflect those of the European Commission. 


\section{Overview}

(1) Stochastic uncertainty in fusion plasmas

(2) Pattern recognition in probabilistic spaces

(3) Geodesic least squares regression

(4) Application in fusion science: edge-localized plasma instabilities

(5) Application in astronomy: Tully-Fisher scaling

(6) Conclusion 


\section{Overview}

(1) Stochastic uncertainty in fusion plasmas

(2) Pattern recognition in probabilistic spaces

(3) Geodesic least squares regression

(4) Application in fusion science: edge-localized plasma instabilities

(5) Application in astronomy: Tully-Fisher scaling

(6) Conclusion 


\section{Fusion energy}

- 'Star on earth'

- Clean, safe, inexhaustible energy source

- Magnetic confinement fusion: tokamak, stellarator, ...

- Confine hot hydrogen isotope plasma with magnetic fields

- ITER: next-generation international tokamak

- Complex physical system, turbulent transport

- Difficult to probe $\rightarrow$ uncertainty in measurements and models
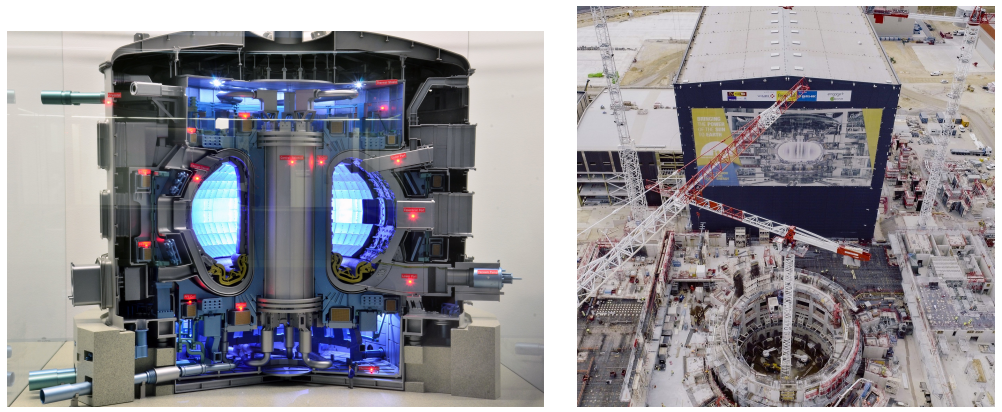


\section{Uncertainty in fusion plasmas}

- Sources of statistical uncertainty:

- Fluctuation of system properties

- Measurement noise
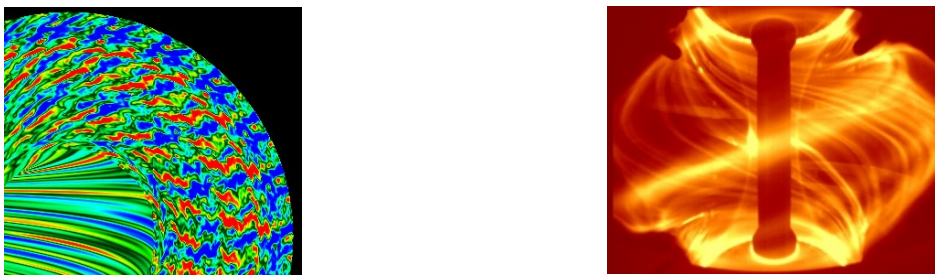

Plasma turbulence (PPPL)

Edge-localized modes (MAST)

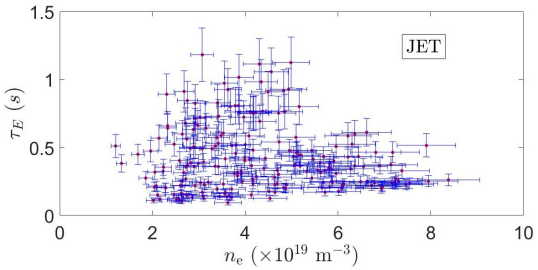




\section{Overview}

(1) Stochastic uncertainty in fusion plasmas

(2) Pattern recognition in probabilistic spaces

(3) Geodesic least squares regression

(4) Application in fusion science: edge-localized plasma instabilities

(5) Application in astronomy: Tully-Fisher scaling

(6) Conclusion 


\section{Difference/distance between points}

Patterns $\leftrightarrow$ distances 


$$
\infty a
$$




\section{Mahalanobis distance}

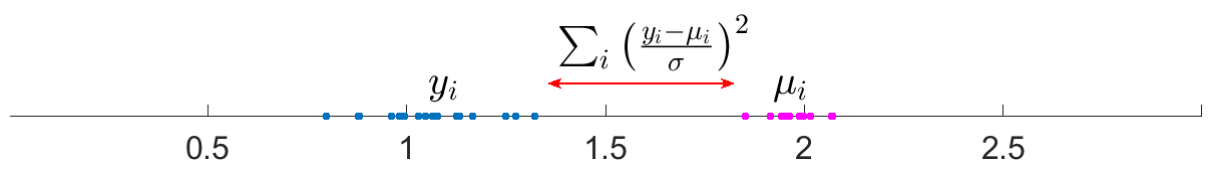




\section{Information geometry}

- Family of probability distributions $\rightarrow$ differentiable manifold

- Parameters $=$ coordinates

- Metric tensor: Fisher information matrix

Parametric probability model: $p(\boldsymbol{x} \mid \boldsymbol{\theta}) \Longrightarrow$

$$
g_{\mu \nu}(\boldsymbol{\theta})=-\mathbb{E}\left[\frac{\partial^{2}}{\partial \theta^{\mu} \partial \theta^{\nu}} \ln p(\boldsymbol{x} \mid \boldsymbol{\theta})\right], \quad \mu, v=1, \ldots, m
$$

$\boldsymbol{\theta}=m$-dimensional parameter vector

- Line element:

$$
\mathrm{d} s^{2}=g_{\mu \nu} \mathrm{d} \theta^{\mu} \mathrm{d} \theta^{v}
$$

- Minimum-length curve: geodesic

- Rao geodesic distance (GD) 


\section{Pattern recognition in probabilistic spaces}

- Pattern recognition:

- Classification, clustering

- Regression analysis

- Dimensionality reduction, visualization

- Observation/prediction (structureless number)

$\rightarrow$ distribution (structured object)

- More information, more flexibility

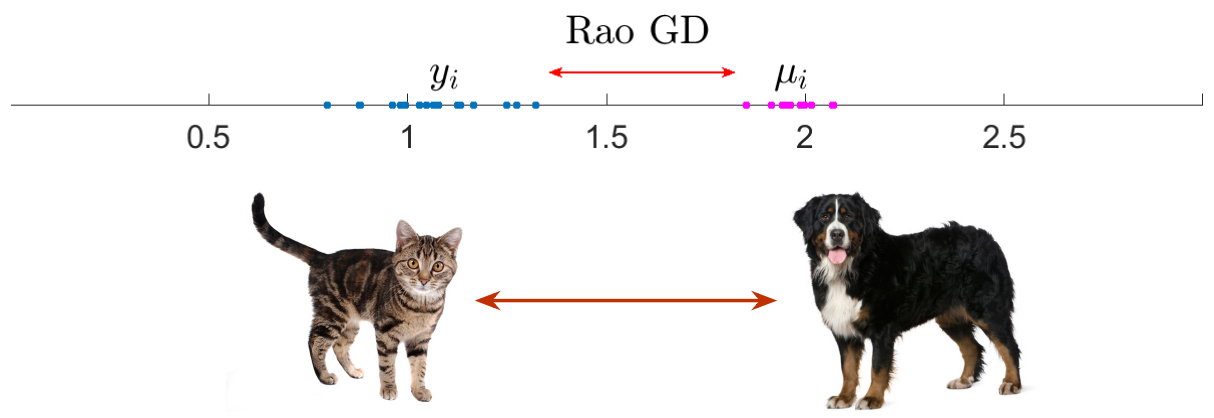




\section{The univariate Gaussian manifold}

- PDF:

$$
p(x \mid \mu, \sigma)=\frac{1}{\sqrt{2 \pi} \sigma} \exp \left[-\frac{(x-\mu)^{2}}{2 \sigma^{2}}\right]
$$

- Line element:

$$
\mathrm{d} s^{2}=\frac{\mathrm{d} \mu^{2}}{\sigma^{2}}+2 \frac{\mathrm{d} \sigma^{2}}{\sigma^{2}}
$$

- Hyperbolic geometry: Poincaré half-plane, Poincaré disk, Klein disk, ...

- Analytic geodesic distance

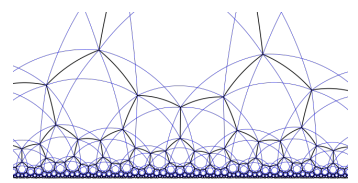

https://www . youtube.com/watch?v=i9IUzNxeH4o 


\section{The pseudosphere (tractroid)}

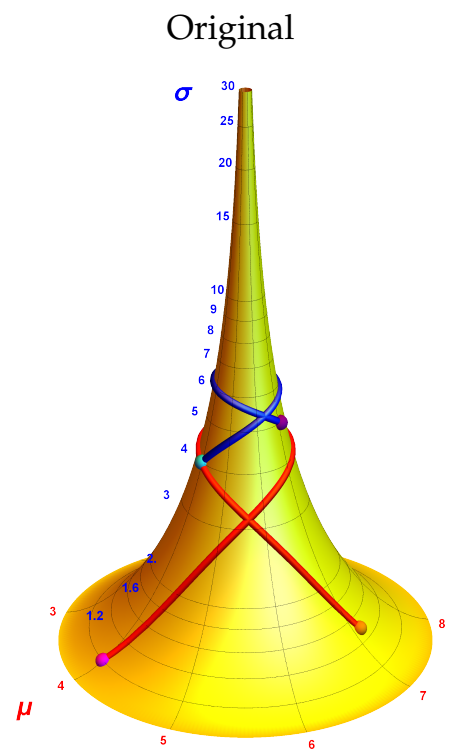

\section{Compressed}

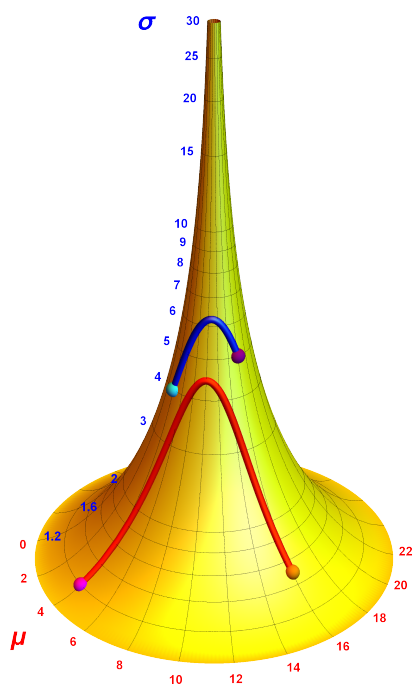




\section{Geodesics on the Gaussian manifold}
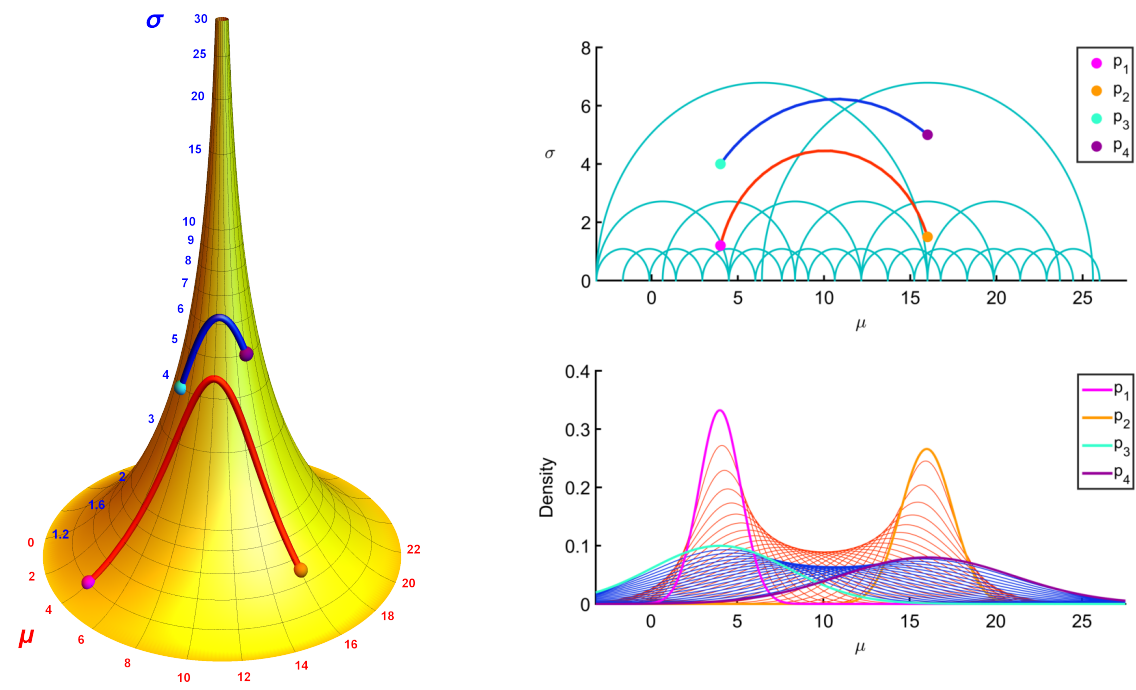


\title{
Data visualization with uncertainty
}

Plasma energy confinement time w.r.t. global plasma parameters

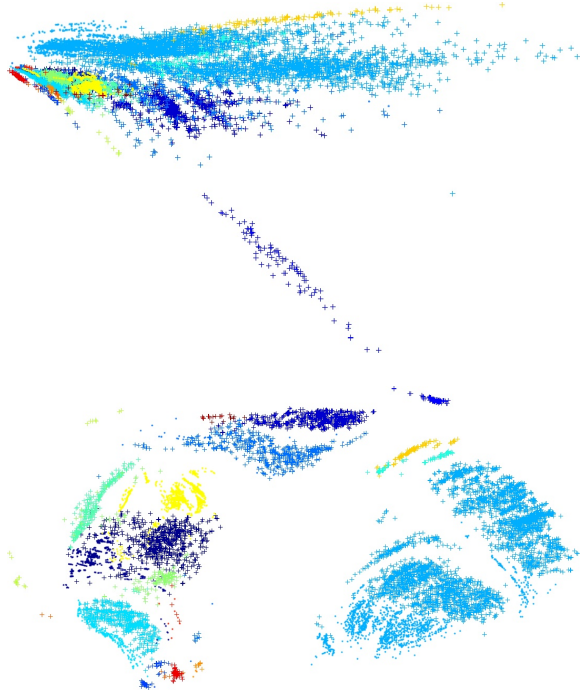

\author{
Euclidean
}

Geodesic 


\section{Overview}

(1) Stochastic uncertainty in fusion plasmas

(2) Pattern recognition in probabilistic spaces

(3) Geodesic least squares regression

(4) Application in fusion science: edge-localized plasma instabilities

(5) Application in astronomy: Tully-Fisher scaling

(6) Conclusion 


\section{Challenges in regression analysis}

- Data uncertainty: measurement error, fluctuations, ...

- Model uncertainty: missing variables, linear vs. nonlinear, Gaussian vs. non-Gaussian, ...

- Heterogeneous data and error bars

- Uncertainty on response $(y)$ and predictor $\left(x_{j}\right)$ variables

- Atypical observations (outliers)

- Near-collinearity of predictor variables

- Data transformations, e.g.

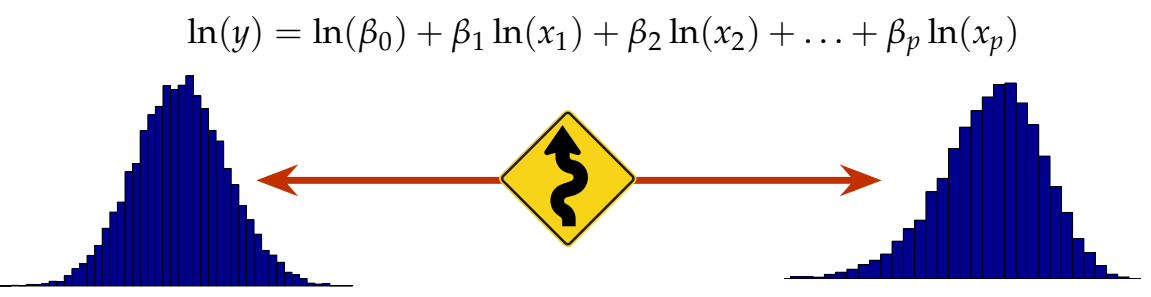




\section{Least squares and maximum a posteriori}

- Workhorse: ordinary least squares (OLS)

- Maximum likelihood (ML) / maximum a posteriori (MAP):

$$
\begin{gathered}
p\left(y_{i} \mid x_{i}, \boldsymbol{\theta}\right)=\frac{1}{\sqrt{2 \pi} \sigma} \exp \left[-\frac{1}{2}\left(\frac{y_{i}-\mu_{i}}{\sigma}\right)^{2}\right] \\
\mu_{i}=f_{i}\left(x_{i}, \boldsymbol{\theta}\right) \stackrel{\text { e.g. }}{=} \beta_{0}+\beta_{1} x_{i}
\end{gathered}
$$

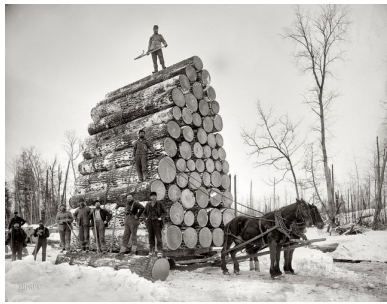

Michigan, circa 1890s.

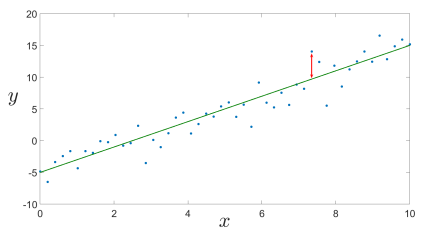

Expected $\leftrightarrow$ Measured 


\section{The minimum distance approach}

- Minimum distance estimation (Wolfowitz, 1952):

Which distribution does the model predict?

Vs.

Which distribution do you observe?

- Gaussian case: different means and standard deviations

- Hellinger divergence (Beran, 1977)

- Empirical distribution: kernel density estimate 


\section{Modeled and observed distribution}
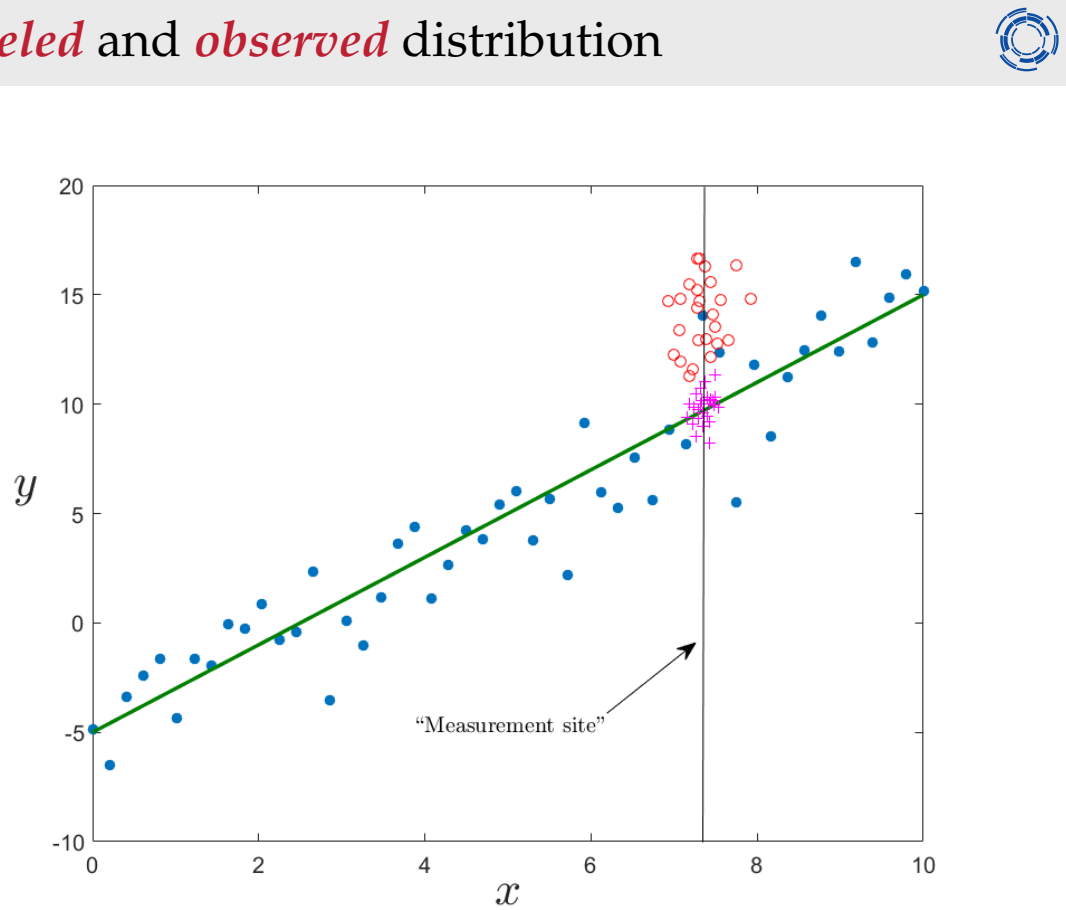


\section{Example: fluid turbulence}
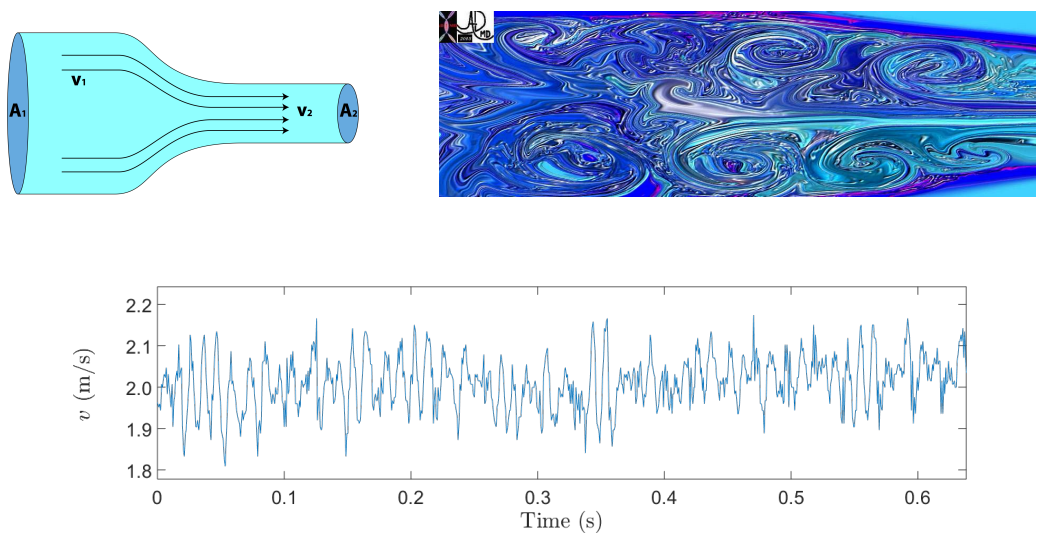


\section{Geodesic least squares}

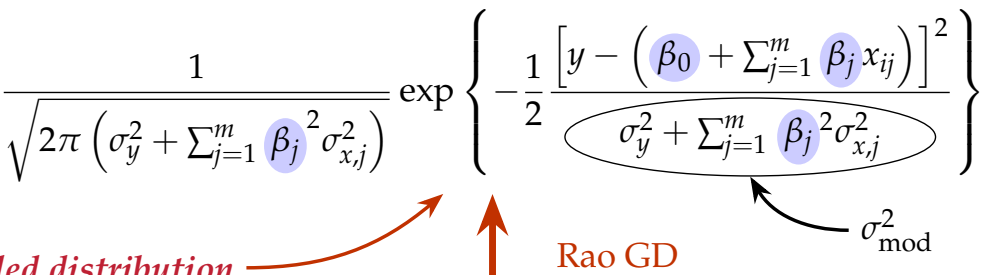

Modeled distribution

$$
\frac{1}{\sqrt{2 \pi} \sigma_{\mathrm{obs}}} \exp \left[-\frac{1}{2} \frac{\left(y-y_{i}\right)^{2}}{\sigma_{\mathrm{obs}}{ }^{2}}\right]
$$

Observed distribution

- Model-based approach: regression on probabilistic manifold

- To be estimated: $\sigma_{\text {obs }}, \beta_{0}, \beta_{1}, \ldots, \beta_{m}$

- iid data: minimize sum of squared GDs

$\Longrightarrow$ geodesic least squares (GLS) regression

- If $\sigma_{\mathrm{mod}}=\sigma_{\mathrm{obs}} \rightarrow$ Mahalanobis distance 


\section{Overview}

(1) Stochastic uncertainty in fusion plasmas

(2) Pattern recognition in probabilistic spaces

(3) Geodesic least squares regression

(4) Application in fusion science: edge-localized plasma instabilities 


\section{Edge-localized modes (ELMs)}

- Repetitive instabilities in plasma edge

- Magnetohydrodynamic origin

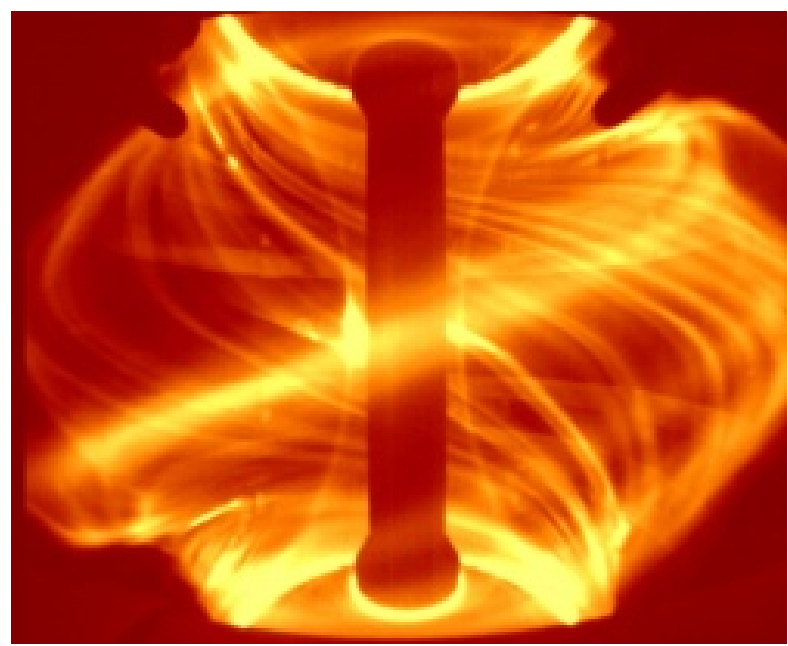

MAST, Culham Centre for Fusion Energy, UK 


\section{Analogy 1: Solar flares}

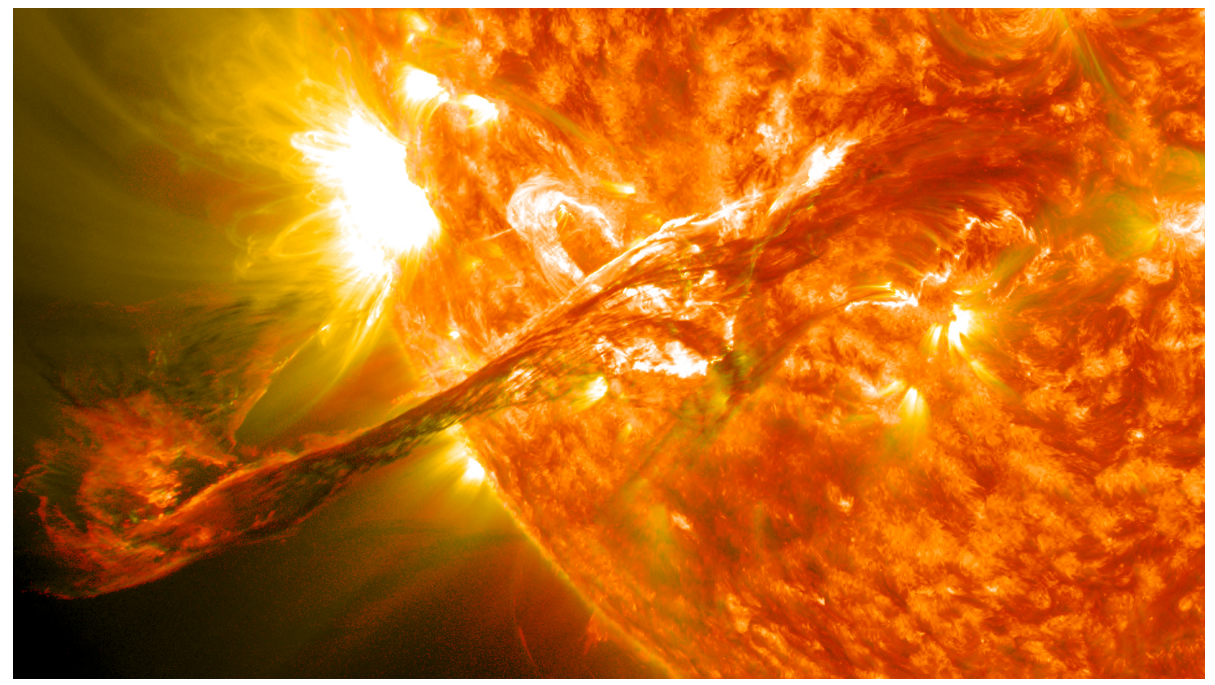




\section{Analogy 2: Cooking pot}

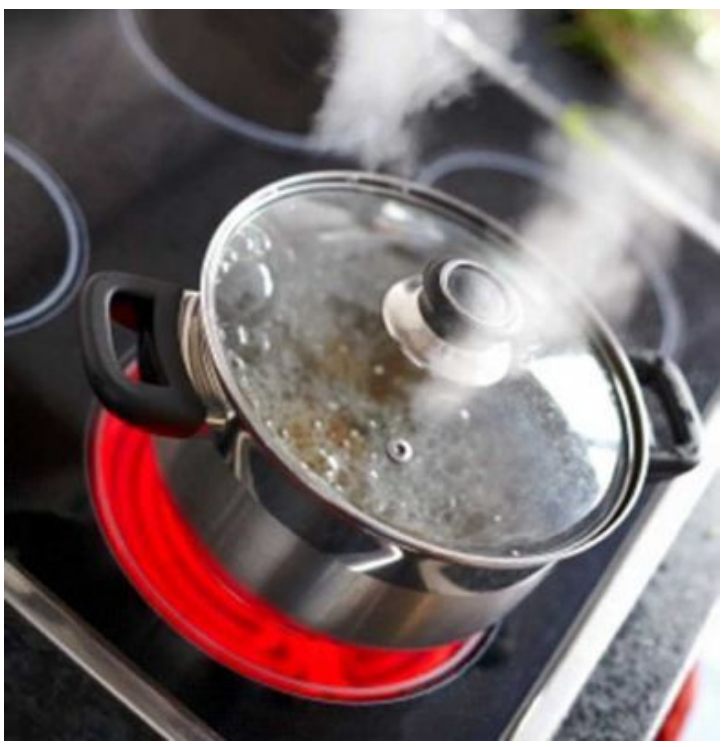




\section{Importance of ELMs}

- Confinement loss

- Potential damaging effects

- Impurity outflux

- $\rightarrow$ ELM control/mitigation

- Energy $\propto(\text { frequency })^{-1}$

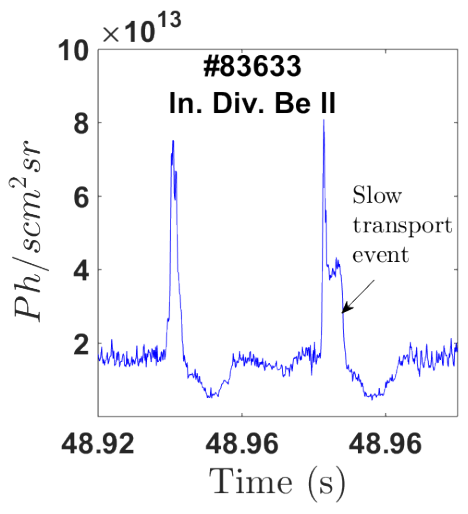




\section{Data extraction: waiting times}

- 32 recent JET discharges

- Waiting time: time before ELM burst

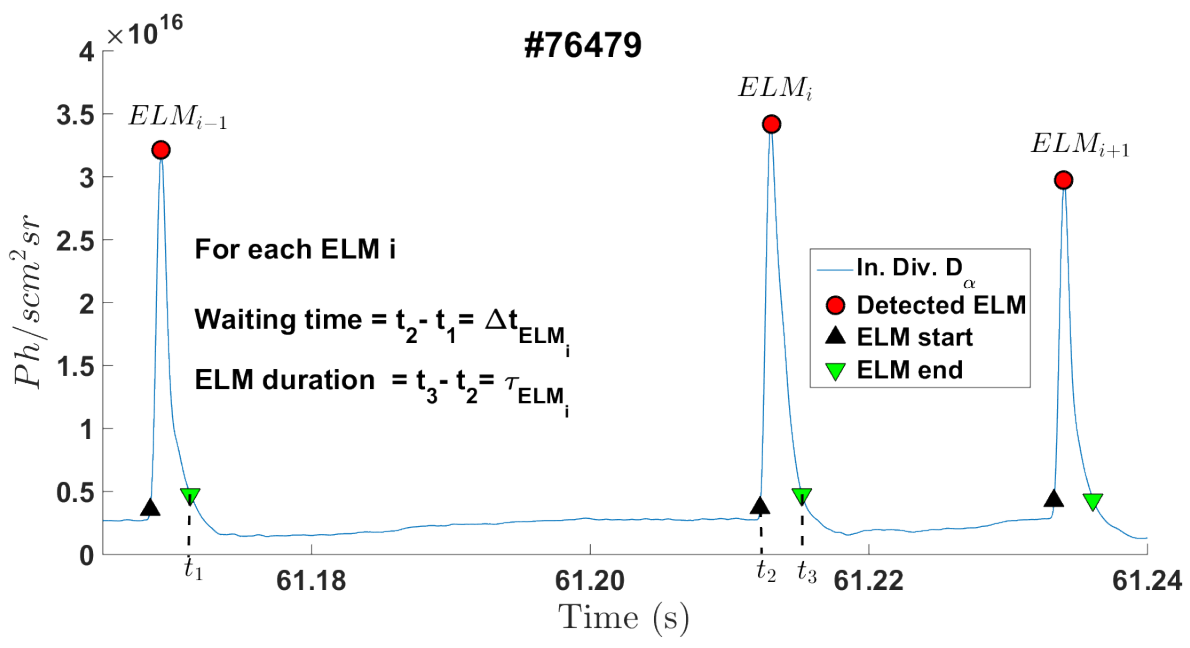




\section{Data extraction: energies}

- Energy carried from the plasma by an ELM

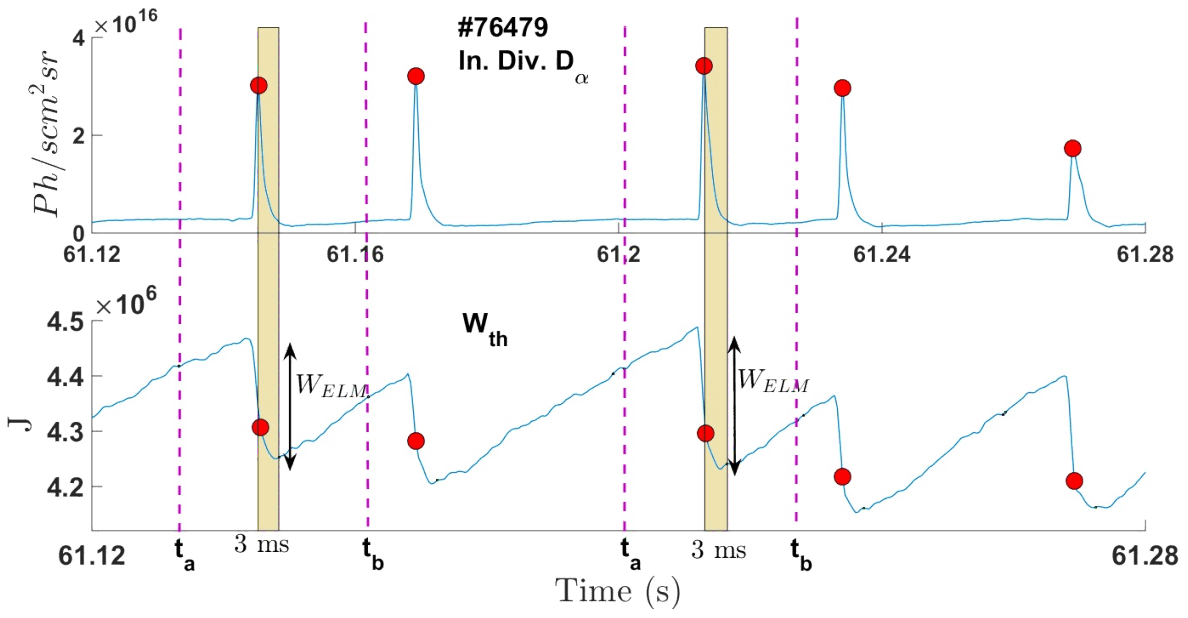




\section{Average waiting times and energies}

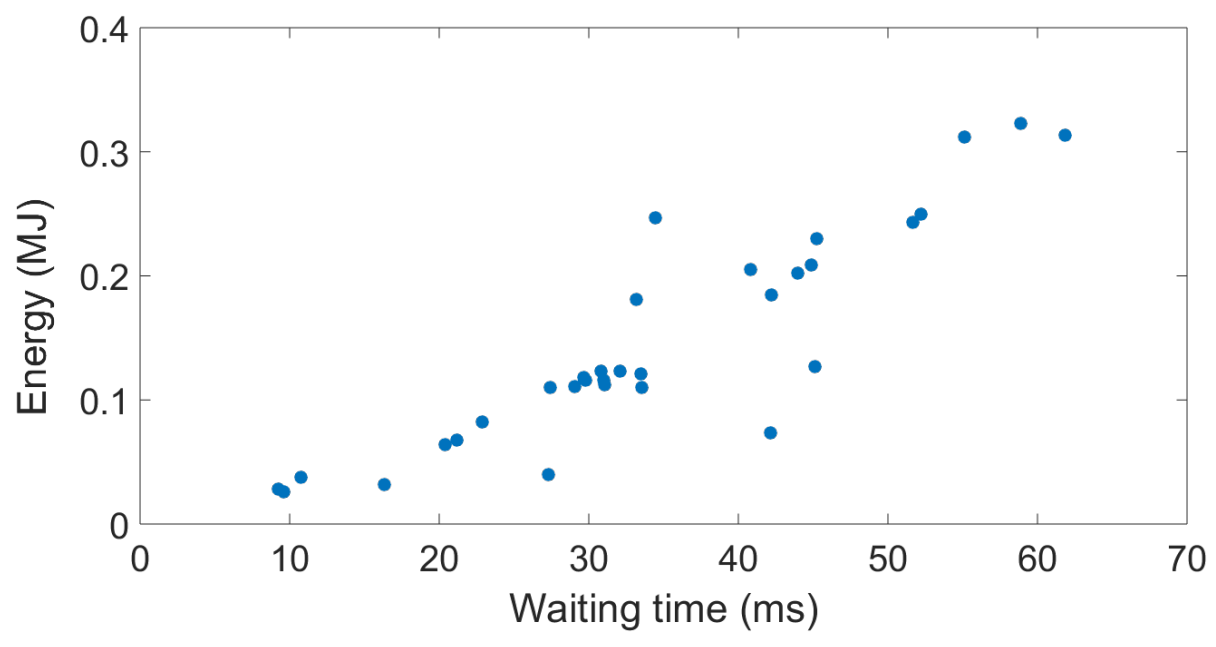




\section{Error bars on averages}

- Standard deviation / $\sqrt{n} \rightarrow$ error bars

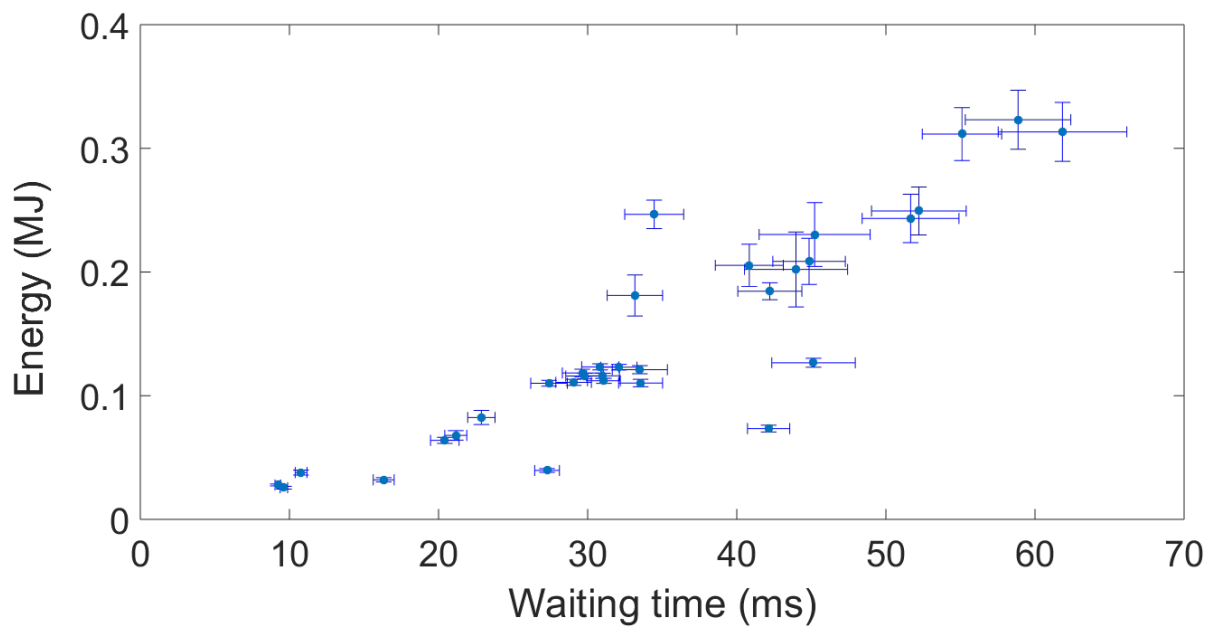




\section{Regression on averages}

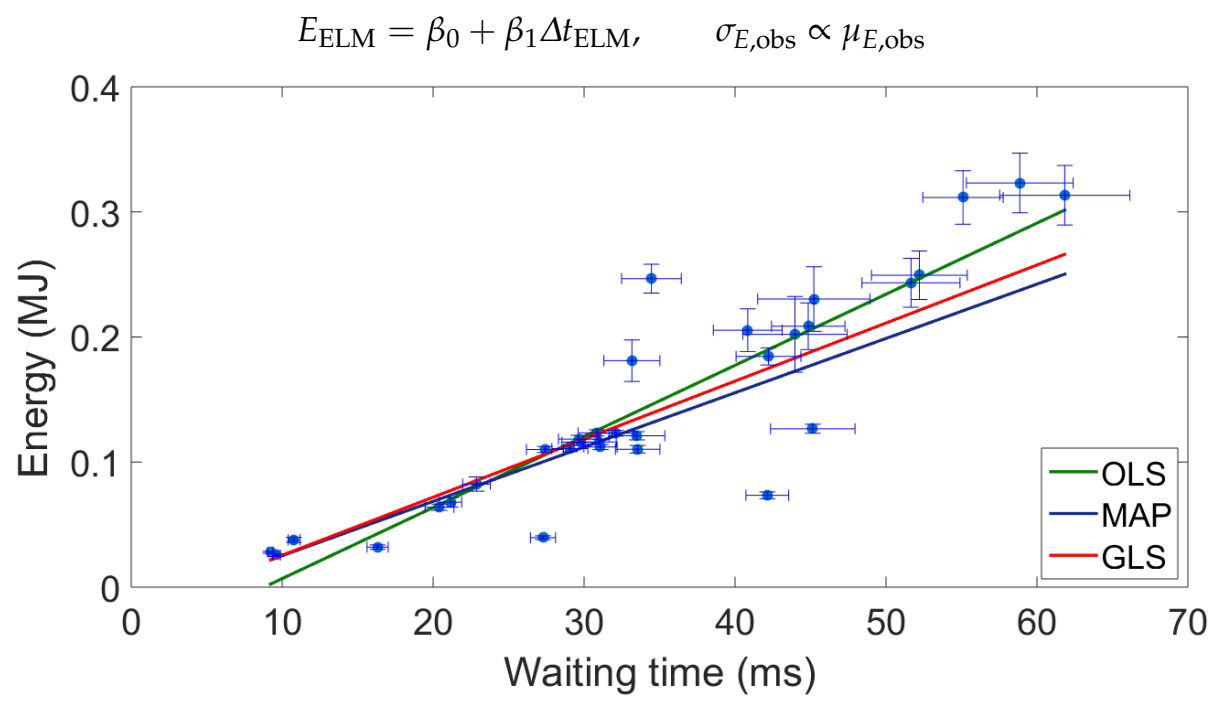


Regression results on pseudosphere

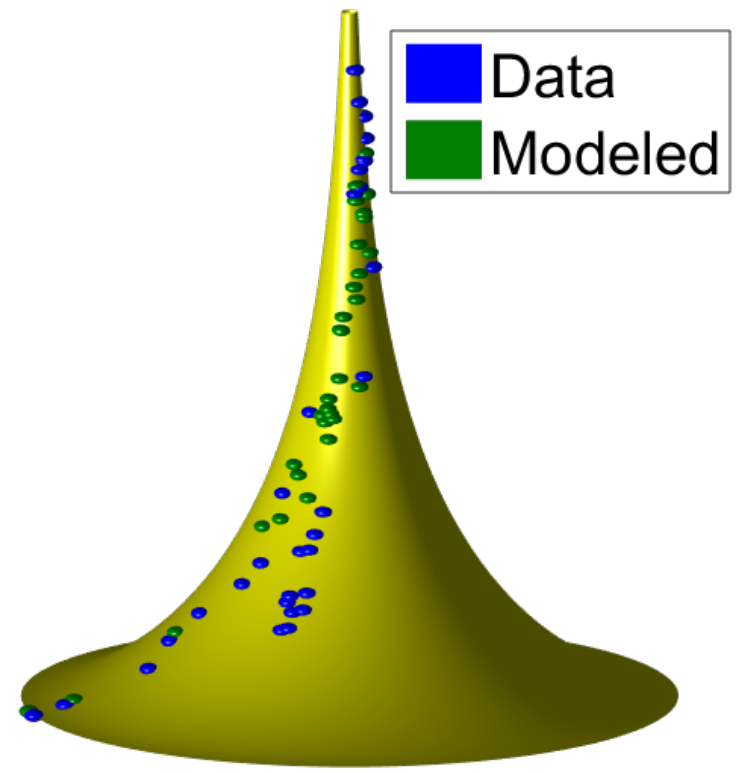




\section{Projected regression results}

Multidimensional scaling:

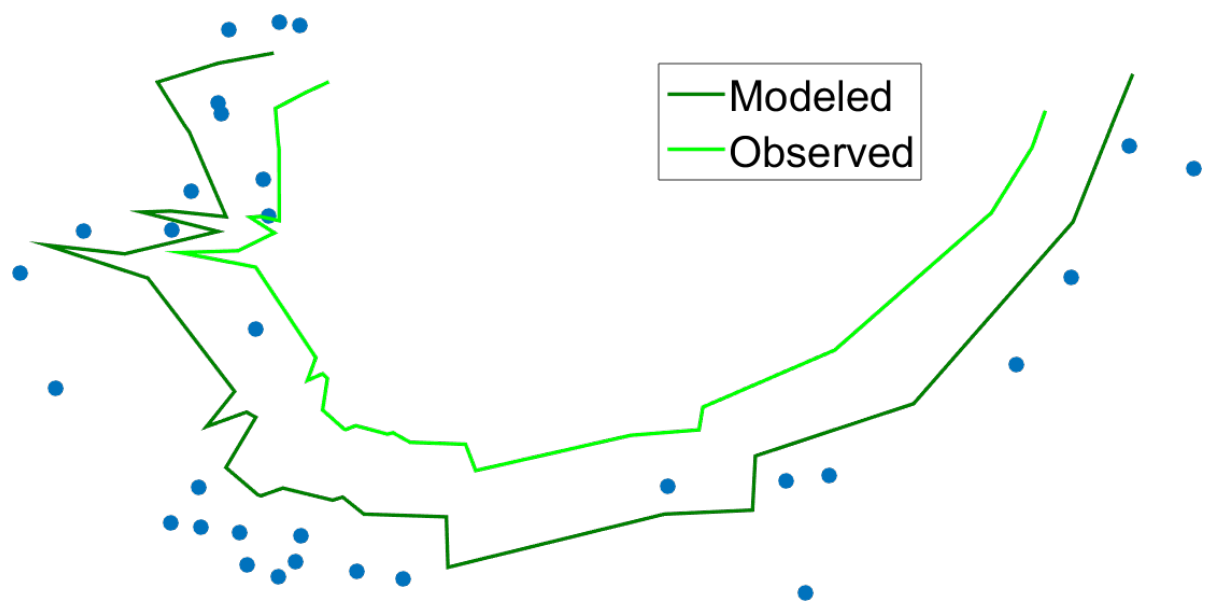




\section{Average vs. collective trend}

Average

\begin{tabular}{lcc}
\hline Method & $\beta_{0}(\mathrm{MJ})$ & $\beta_{1}(\mathrm{MJ} / \mathrm{s})$ \\
\hline OLS & -0.050 & 5.7 \\
GLS & -0.021 & 4.6 \\
\hline
\end{tabular}

Individual

\begin{tabular}{lcc}
\hline Method & $\beta_{0}(\mathrm{MJ})$ & $\beta_{1}(\mathrm{MJ} / \mathrm{s})$ \\
\hline OLS & 0.024 & 3.2 \\
GLS & -0.022 & 4.2 \\
\hline
\end{tabular}

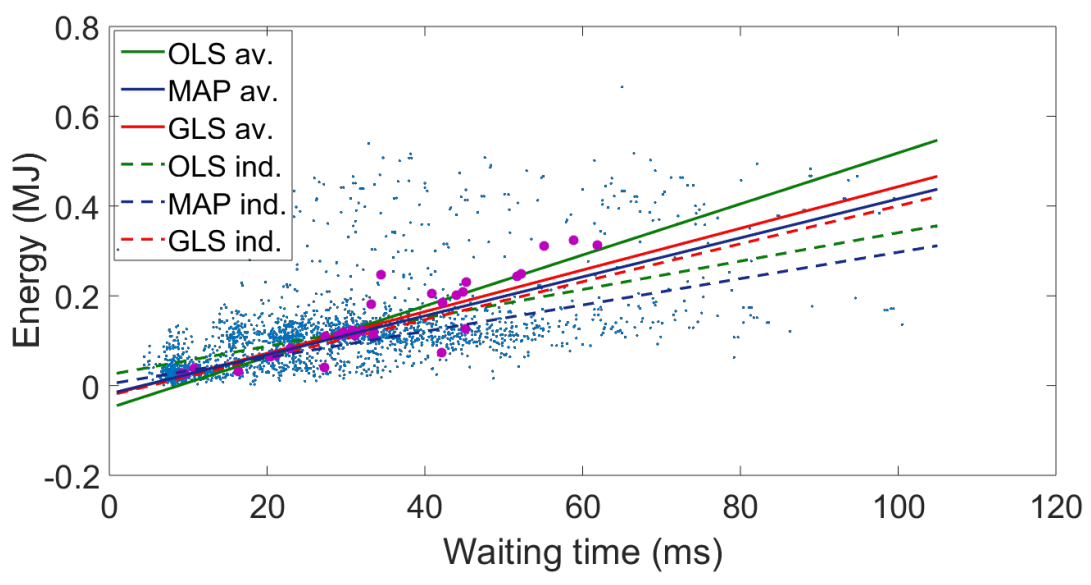




\section{Overview}

(1) Stochastic uncertainty in fusion plasmas

(2) Pattern recognition in probabilistic spaces

(3) Geodesic least squares regression

(4) Application in fusion science: edge-localized plasma instabilities

(5) Application in astronomy: Tully-Fisher scaling 


\section{Baryonic Tully-Fisher Relation (BTFR)}

- Simple, tight relation for disk galaxies:

$$
M_{b}=\beta_{0} V_{f}^{\beta_{1}} \quad\left\{\begin{array}{l}
M_{b}=\text { total (stellar }+ \text { gaseous) baryonic mass }\left(M_{\odot}\right) \\
V_{f}=\text { rotational velocity }\left(\mathrm{km} \mathrm{s}^{-1}\right)
\end{array}\right.
$$

- Various purposes:

- Distance indicator

- Constraints on galaxy formation models

- Test for alternatives to $\Lambda \mathrm{CDM}$ cosmological model (slope and scatter)

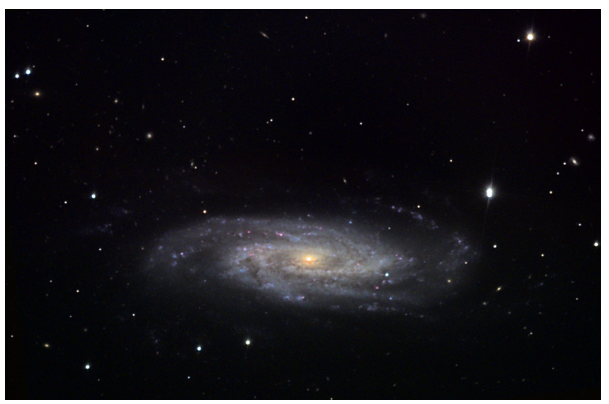




\section{Experiments}

- 47 gas-rich galaxies (McGaugh, Astron. J. 143, 40, 2012)

- Loglinear $\left(\sigma_{\mathrm{obs}, i} \equiv s_{\mathrm{obs}}\right)$ and nonlinear $\left(\sigma_{\mathrm{obs}, i}=r_{\mathrm{obs}} M_{b}\right)$

- Benchmarking:

- Ordinary least squares (OLS)

- Bayesian: errors in all variables, marginalized standard deviations (Bayes)

- Geodesic least squares (GLS)

- Kullback-Leibler least squares (KLS) 


\section{Loglinear regression}

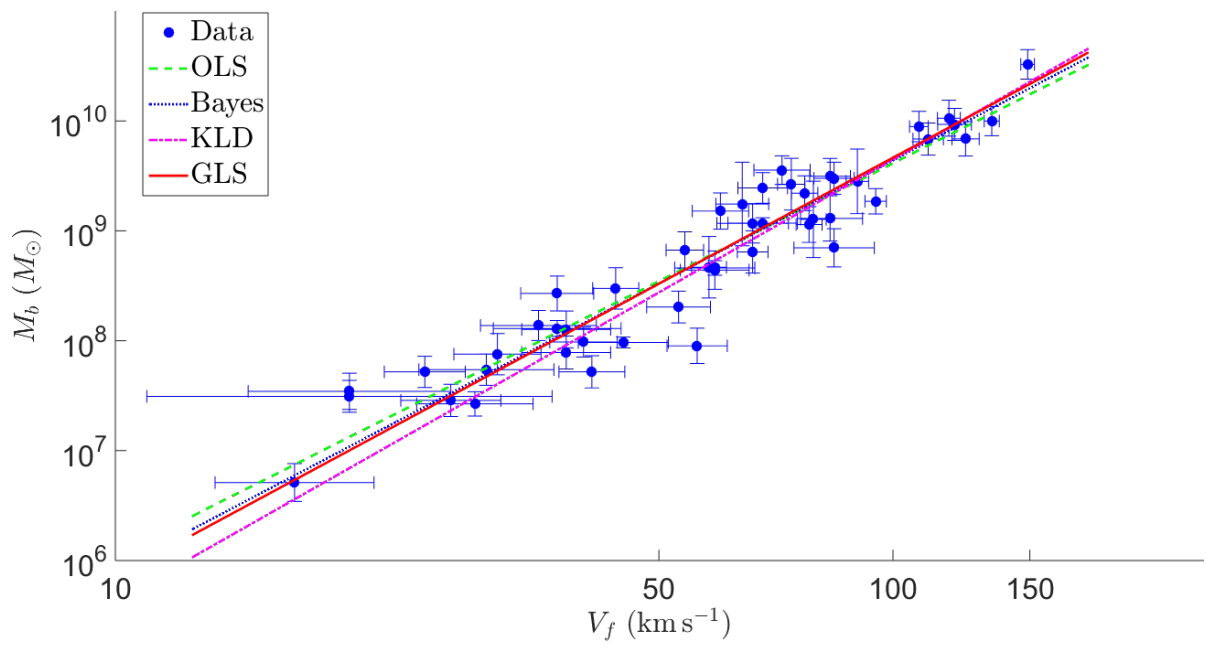




\section{Nonlinear regression}

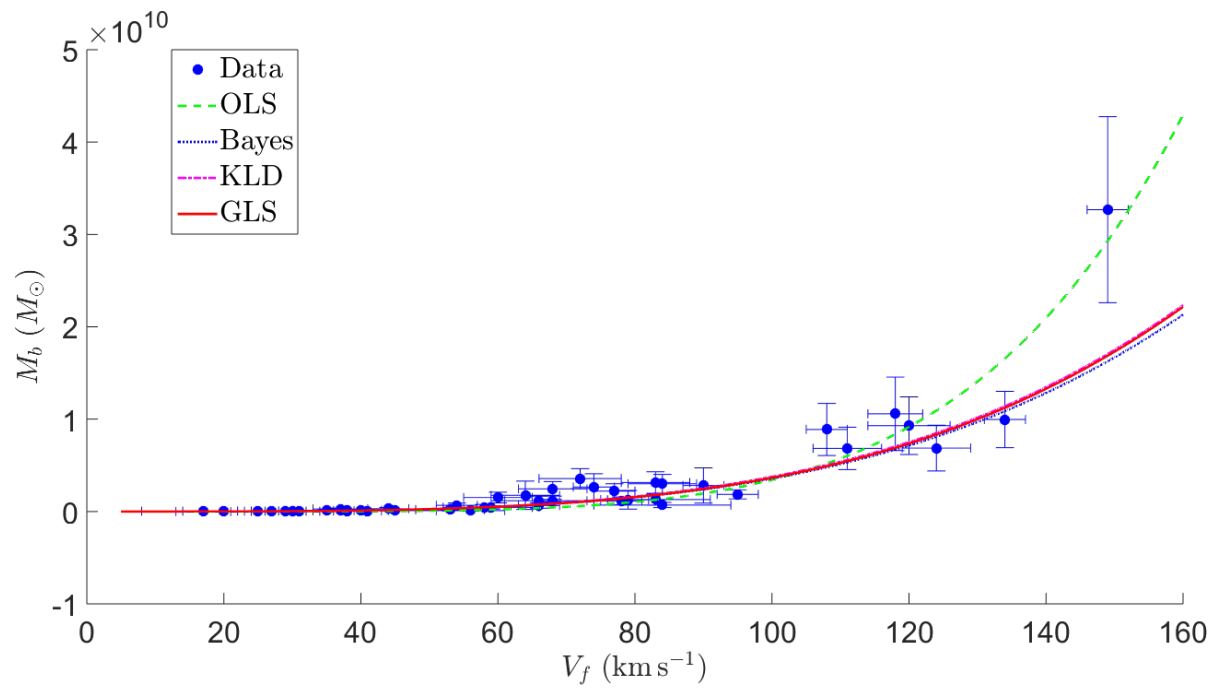




\section{Parameter distributions}
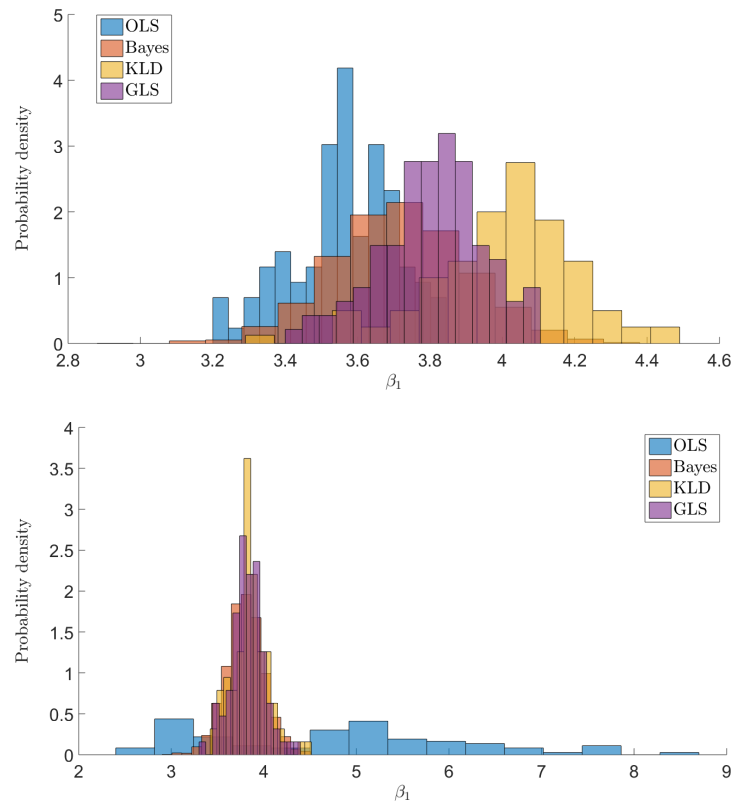


\section{GLS uncertainty estimates}

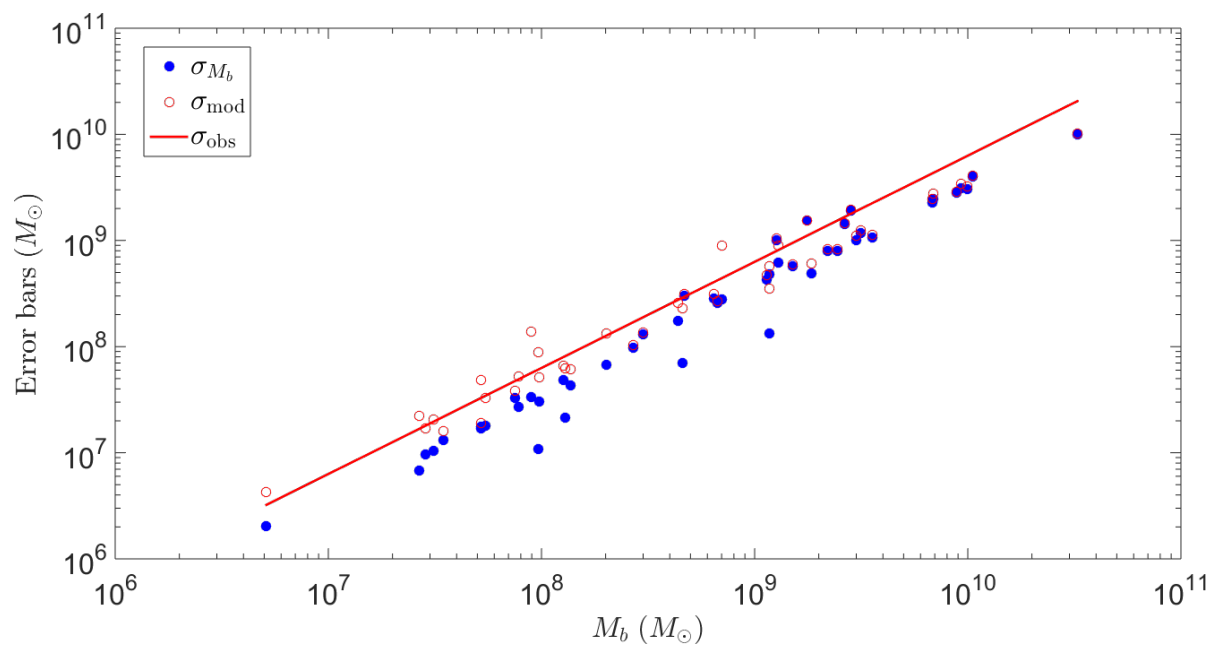

$r_{M_{b}} \approx 38 \%, r_{\mathrm{obs}} \approx 63 \%$ 


\section{Interpretation on pseudosphere}

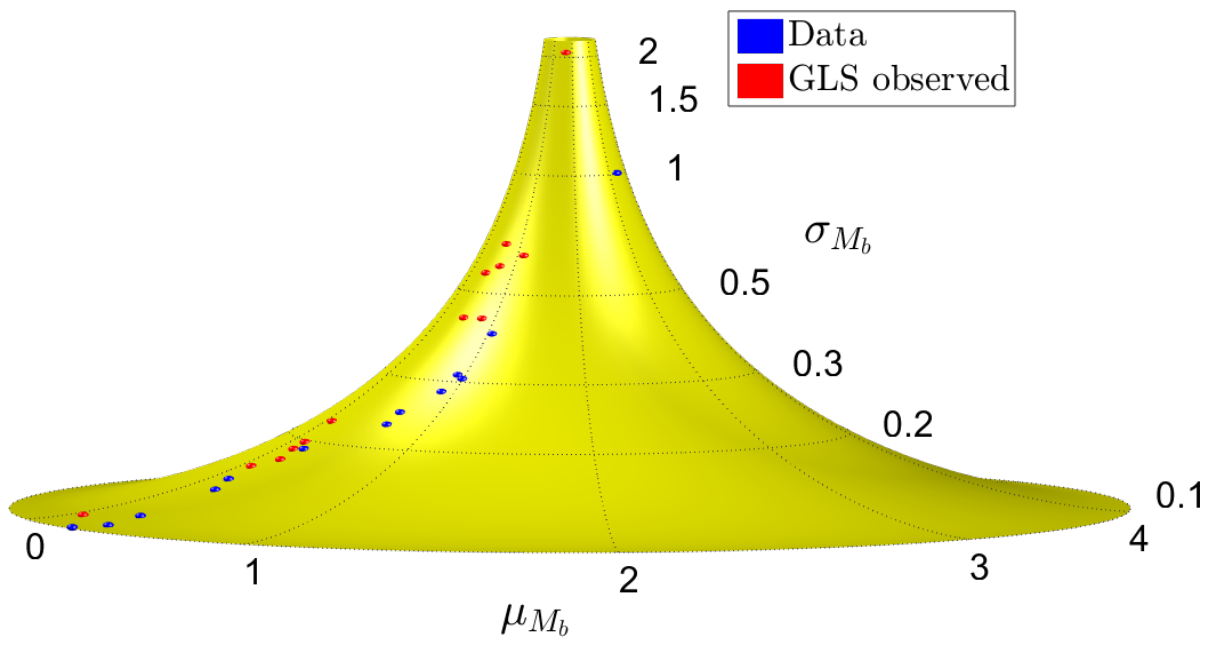




\section{Overview}

(1) Stochastic uncertainty in fusion plasmas

(2) Pattern recognition in probabilistic spaces

(3) Geodesic least squares regression

(4) Application in fusion science: edge-localized plasma instabilities

(5) Application in astronomy: Tully-Fisher scaling

(6) Conclusion 


\section{Conclusions}

- Probabilistic modeling of stochastic system properties

- Information geometry: distance measure, geometrical intuition

- Pattern recognition in probabilistic spaces

- More information, more flexibility

- Geodesic least squares regression: flexible and robust

- Easy to use, fast optimization 